\title{
Anastomosis yeyunal término-terminal con xenoinjerto de pericardio bovino en equinos
}

\author{
END-TO-END JEJUNAL ANASTOMOSIS WITH BOVINE PERICARDIUM XENOGRAFT IN EQUINES
}

Ceesar N. Aguilar G. ${ }^{1,3}$, Alfredo Delgado C. ${ }^{1}$, Alfonso Chavera C. ${ }^{2}$, Medalí Cueva R. ${ }^{1}$

\section{Resumen}

Se investigó la viabilidad del xenoinjerto de pericardio bovino no tratado como biomaterial para el revestimiento de la anastomosis de intestino delgado en equinos a fin de evitar dehiscencias, fugas, fístulas y formación de adherencias en el posoperatorio. Se trabajó con siete equinos sometidos a anastomosis término-terminal yeyunal revestida con xenoinjerto de pericardio bovino no tratado y un grupo control de siete equinos sometidos solamente a anastomosis término-terminal yeyunal. Se evaluó el dolor postoperatorio, los componentes celulares y las características organolépticas del líquido peritoneal. Se encontró que el revestimiento con xenoinjerto de pericardio bovino no genera diferencias significativas en la cuantificación del dolor (cólico posoperatorio) ni en el recuento total de células nucleadas del líquido peritoneal, ni influye en las alteraciones del color, turbidez y olor del líquido peritoneal, demostrando que el xenoinjerto de pericardio bovino sin tratamiento como revestimiento de la anastomosis yeyunal no influye en las funciones del sistema alimentario desde el punto de vista del exámen clínico posoperatorio de campo. Se concluye que el xenoinjerto de pericardio bovino no tratado es viable y efectivo porque refuerza el proceso de remodelación cicatricial, evitando posibles fugas anastomóticas y peritonitis.

Palabras clave: equino; pericardio bovino; anastomosis; xenoinjerto; enterectomía; intestino delgado; cólico posoperatorio; dolor postoperatorio

\section{AbSTRACT}

The feasibility of untreated bovine pericardium xenograft as biomaterial for the lining of the small intestine anastomosis in horses to avoid dehiscences, leakage, fistulas and formation of adhesions in the postoperative period was investigated. Seven horses were submitted to end-to-end jejunal anastomosis coated with untreated bovine pericardial xenograft and a control group of seven horses submitted to end-to-end jejunal anasto-

\footnotetext{
${ }^{1}$ Clínica de Animales Mayores, ${ }^{2}$ Laboratorio de Histopatología, Embriología y Patología Animal, Facultad de Medicina Veterinaria, Universidad Nacional Mayor de San Marcos, Lima, Perú

${ }^{3}$ Email: ceniague@gmail.com
}

Recibido: 7 de junio de 2017

Aceptado para publicación: 28 de septiembre de 2017 
mosis only. Postoperative pain, cellular components and organoleptic characteristics of the peritoneal fluid were evaluated. It was found that the bovine pericardium xenograft coating does not generate significant differences in the quantification of pain (postoperative colic) nor in the total nucleated cell count of the peritoneal fluid nor does it influence the alterations of the color, turbidity and smell of the peritoneal fluid, demonstrating that untreated bovine pericardial xenograft as a covariant of the jejunal anastomosis does not influence the functions of the alimentary system from the point of view of the postoperative clinical examination. It is concluded that untreated bovine pericardium xenograft is viable and effective because it reinforces the cicatricial remodeling process, avoiding possible anastomotic leakage and peritonitis

Key words: equine; bovine pericardium; anastomosis; xenograft; enterectomy; small intestine; post-surgery colic; post-surgery pain

\section{INTRODUCCIÓN}

El cólico es una de las principales causas de muerte en los caballos y tiene un impacto económico significativo en su crianza (Abutarbush et al., 2005), donde los problemas del intestino delgado, en general, tienen peor pronóstico que los que involucran al intestino grueso (van den Boom y van der Velden et al., 2001). Las afecciones del intestino delgado pueden representar del 25 al $64 \%$ de los casos de cólicos tratados en los hospitales veterinarios, donde el 58-85\% son causados por lesiones estrangulares y el resto por obstrucciones no estrangulares (Freeman, 2012).

La resección y anastomosis del intestino delgado se realiza generalmente en caballos con malestar abdominal asociado a patologías obstructivas (Stewart et al., 2014). Es necesaria una pronta intervención quirúrgica antes de que la condición del cólico progrese a posible disfunción multiorgánica (Schwarz et al., 2012), mientras que una laparotomía exploratoria retardada puede resultar en la ruptura visceral o deterioro de la condición del paciente (Marshall y Blikslager, 2012; Smith et al., 2005). La falla anastomótica gastrointestinal se debe a dehiscencia, fugas y fístulas, las cuales son complicaciones importantes en las cirugías (Telem et al., 2010; Testini et al., 2014).
Los intentos para mejorar el resultado quirúrgico en el equino deben dirigirse a un manejo cuidadoso del intestino durante la cirugía y a la prevención del íleo paralítico posoperatorio para evitar la formación de adherencias (van de Boom y van der Velden, 2001), que son la causa más frecuente de cólico posoperatorio y la segunda causa más común de laparotomía repetida; asimismo, el pronóstico para los caballos sometidos a laparotomía repetida debido a adherencias es pobre, con tasas de supervivencia reportadas de 0 a 20\% (Alonso et al., 2014). Las adherencias son causa frecuente de frustración para los cirujanos, lo que estimula la investigación de productos y métodos para prevenir su formación (Claunch y Mueller, 2012).

Las nuevas técnicas quirúrgicas proponen el uso de bioprótesis para evitar fugas, fístulas y adherencias (Testini et al., 2014), habiendo actualmente disponibilidad de parches de pericardio bovino tratados con glutaraldehído para evitar el trasplante de proteínas y de ADN bovino en el huésped, pero con el inconveniente que podrían calcificarse e incluso adquirir un comportamiento citotóxico (Li et al., 2014). Por otro lado, la liofilización del pericardio bovino como una medida de tratamiento produce daños en las fibras de colágeno por la pérdida de agua, implicando una drástica disminución de sus propiedades mecánicas, por lo que no se 
garantiza su uso (Polak y Pitombo, 2011). La aplicación de una malla sintética o parches de serosa yeyunal han sido reportadas como eficaces en el tratamiento quirúrgico de las lesiones intestinales graves (Astarcioçlu et al., 2001). Otras técnicas quirúrgicas suplementarias reportadas en varias especies animales incluyen parche de serosa, envoltura omental, malla de ácido poliglicólico, envoltura con injerto peritoneal y la aplicación de sellador de fibrina, pero con poca evidencia objetiva sobre el soporte mecánico o de protección contra fugas postoperatorias (Hansen y Monnet, 2013).

El pericardio bovino tratado con glutaraldehído ha sido usado para parchar la línea de sutura de la anastomosis yeyunal en cerdos, modelo quirúrgico experimental, con miras a mejorar la técnica quirúrgica entérica en medicina humana, el cual debería ser probado de manera experimental en otras especies animales (Testini et al., 2014). Ante la problemática existente, se planteó un estudio en caballos sometidos a enterectomía término-terminal con y sin xenoinjerto de pericardio bovino con el objetivo de cuantificar el dolor pos-anastomosis yeyunal y las variaciones de las concentraciones celulares y organolépticas del líquido peritoneal.

\section{Materiales y Métodos}

El presente estudio se realizó en las instalaciones de la Universidad Nacional de Cajamarca, Perú, bajo condiciones de campo con 14 equinos criollos (machos y hembras) entre 3 y 10 años, procedentes de la campiña de Cajamarca. Los caballos fueron clasificados como ASA 1, según los criterios de la Asociación Americana de Anestesiología (Nazar et al., 2015). Los animales fueron distribuidos en forma aleatoria en dos grupos sometidos a enterectomía términoterminal con (grupo A) y sin (grupo B) xenoinjerto de pericardio bovino, respectivamente.
Los caballos fueron dosificados con Ivermectina al $1.85 \%$ y recibieron alfalfa $a d$ libitum durante 20 días previos a la cirugía.

\section{Líquido Peritoneal}

Se colectó líquido peritoneal de todos los caballos por abdominocentesis en tubos con EDTA (Siobhan, 2014) previo a la cirugía y a los 4 y 8 días de la misma (Mcgowan y Hodgson, 2014) para determinar el recuento total de células nucleadas (RTCN) (Marshall y Blikslager, 2012), utilizando un analizador automatizado para hematología (Rayto RT7600S). Asimismo, se hizo la evaluación de color, turbidez y olor del líquido peritoneal, basados en Al-Rukibat et al. (2006), Siobhan (2014) y Sirois (2015).

Los datos fueron evaluados mediante un análisis de varianza factorial para medidas repetidas, encontrando que se cumplía el supuesto de esfericidad de Mauchly; permitiendo a la vez, realizar múltiples comparaciones por pares con el ajuste de Bonferroni, utilizando el programa estadístico SPSS v. 22.

\section{Técnica Anestésica}

Después de un ayuno de 24 h y una restricción de agua de $3 \mathrm{~h}$, se colocó una vía intravenosa (IV) de $\mathrm{NaCl}$ al $0.9 \%$ para la perfusión de los fármacos. La estrategia anestésica empezó con la premedicación de xilazina $(0.5 \mathrm{mg} / \mathrm{kg} \mathrm{IV})$, acepromazina $(0.03 \mathrm{mg} / \mathrm{kg} \mathrm{IV})$ y tramadol (2 mg/kg IV), además de ceftriaxona $(20 \mathrm{mg} / \mathrm{kg} \mathrm{IV})$ como antibiótico profiláctico. La inducción se realizó con diazepam $(0.25 \mathrm{mg} / \mathrm{kg}$ IV) y ketamina $(5 \mathrm{mg} / \mathrm{kg} \mathrm{IV})$. Se procedió con la intubación endotraqueal para el mantenimiento de la anestesia con isofluorano diluido en $\mathrm{O}_{2}$ médico a bajo flujo, a una concentración alveolar mínima (MAC) promedio de $1.4 \%$, manejada según los requerimientos del paciente. Durante la anestesia, se efectuó infusión IV de lidocaína ( $2 \mathrm{mg} / \mathrm{kg}$ durante $10-15 \mathrm{~min}$, seguido de infusión continua de $0.05 \mathrm{mg} / \mathrm{kg} / \mathrm{min}$ ) como agente procinético intestinal (Driessen, 2013; Divers, 2014). 

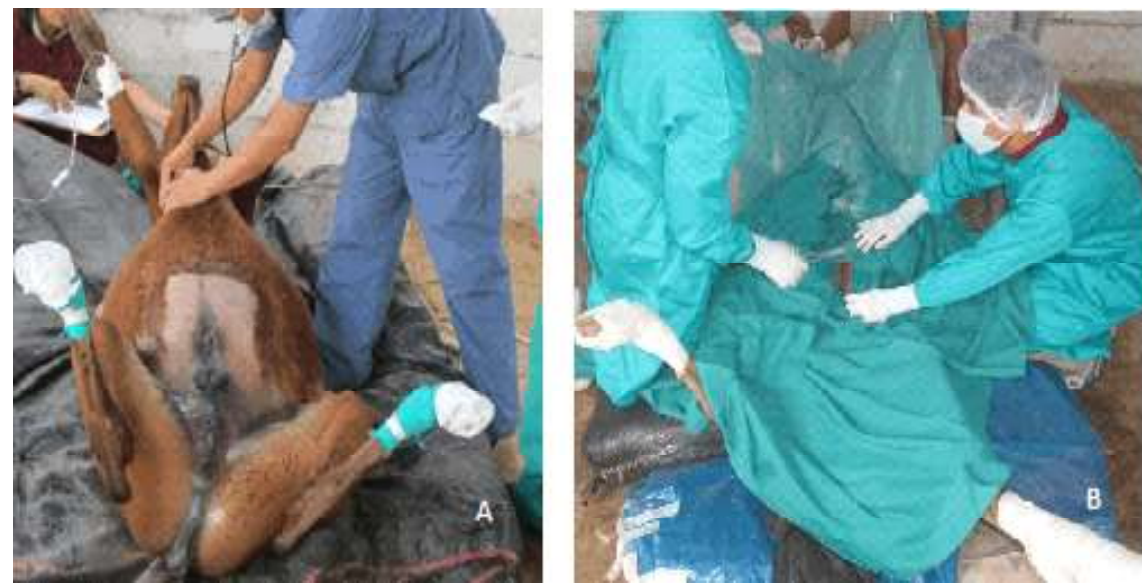

Figura 1. Preparación de la zona quirúrgica. (A) Posicionamiento del paciente, tricotomía y asepsia quirúrgica; B: Ubicación de campos estériles previo a la cirugía

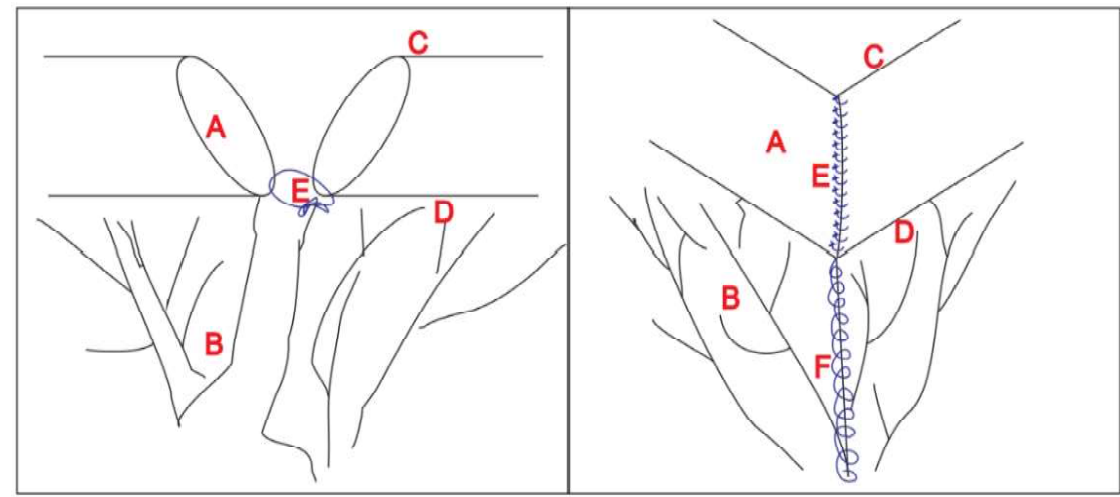

Figura 2. Ilustración del corte experimental del yeyuno, en ángulo aproximado de $50^{\circ}$ para extraer el segmento intestinal y la colocación de las suturas de ácido poliglicólico. (A) Corte del yeyuno; (B) Mesenterio; (C) Zona contramesentérica (termina en conformación de «V»); (D) Zona mesentérica; (E) Sutura simple discontinua en la línea anastomótica; (F) Sutura simple continua del mesenterio (ilustración del autor)

\section{Técnica Quirúrgica}

El abordaje quirúrgico fue a través de una laparotomía ventral medial (Lorello y Orsini, 2014) de 8-12 cm en la línea alba y a la altura del ombligo (Figura 1). Se exteriorizó un asa intestinal yeyunal que se ubicó sobre campos quirúrgicos de tercer orden humedecidos con suero fisiológico al $0.9 \%$. Se consideró ligar la vasculatura yeyunal (König y Liebich, 2005) para luego seccionar cerca de $10 \mathrm{~cm}$ del asa yeyunal con cortes oblícuos en un ángulo de 50 grados (Freeman, 2012). Al término de la enterectomía término-terminal se consideró el cierre del mesenterio con sutura simple continua (Bladon y Hillyer, 2000).

\section{Técnica quirúrgica para el grupo $B$}

La anastomosis término-terminal sin revestimiento de pericardio bovino se realizó con un patrón de sutura simple discontinuo 

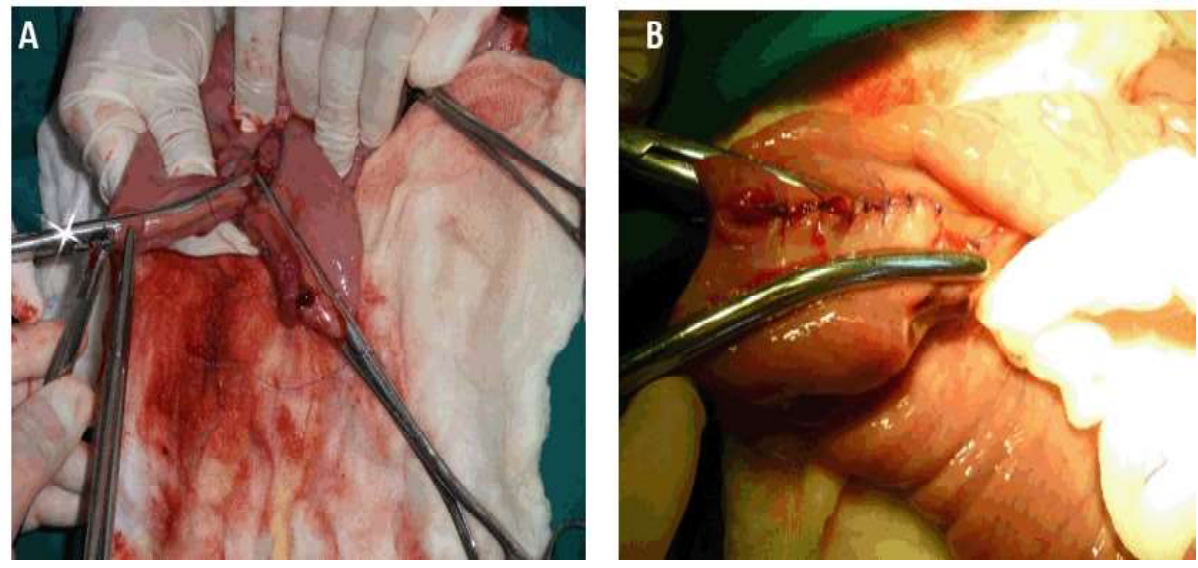

Figura 3. Técnica quirúrgica de anastomosis término-terminal con un solo patrón de sutura. (A) Sutura simple discontinua en la zona contra-mesentérica con ácido poliglicólico N. ${ }^{\circ}$ 3-0, luego de aplicar la primera sutura en la zona mesentérica; (B) Aplicación de las suturas simples discontinuas para completar toda la línea de sutura en la periferia del yeyuno

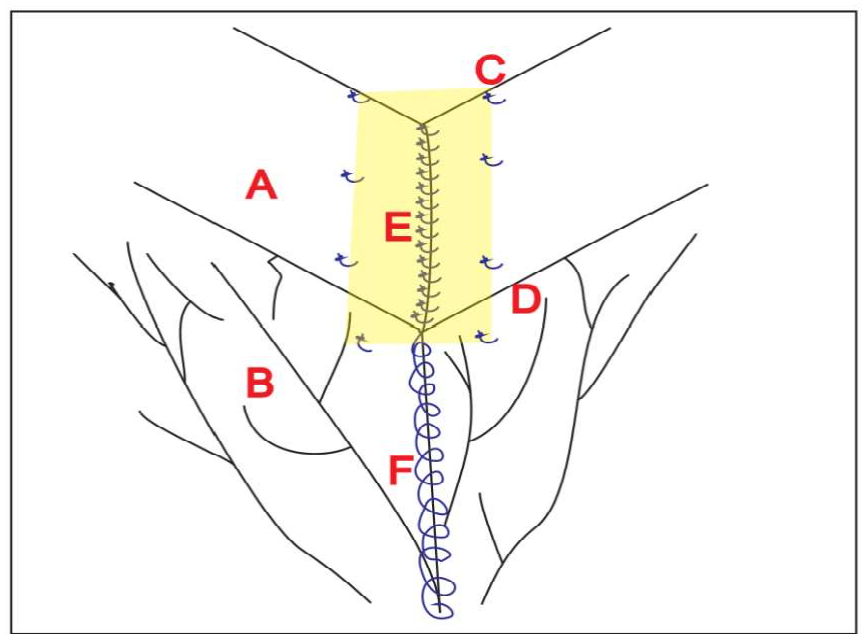

Figura 4. Ilustración del revestimiento con xenoinjerto de pericardio bovino sobre la línea de sutura anastomótica (color amarillento), fijado con suturas simples discontinuas de ácido poliglicólico. (A) Yeyuno; (B) Mesenterio; (C) Zona contramesentérica; (D) Zona mesentérica; (E) Sutura simple discontinua en la línea anastomótica; (F) Sutura simple continua del mesenterio (ilustración del autor)

(Fossum, 2009), con material de sutura absorbible (ácido poliglicólico N. ${ }^{\circ} 3-0$ ), desde la serosa, incluyendo la muscular longitudinal, la muscular circular y la submucosa, basados en las estructuras histológicas reportadas por Gelberg (2014) (Figuras 2 y 3 ), y considerando la evidencia reportada por Freeman (2012), de que la anastomosis en una sola sutura también funciona en el intestino delgado equino. El intestino se devolvió a la cavidad abdominal y se continuó con el cierre de la pared abdominal con un patrón de sutura simple continua en peritoneo y la línea alba, con hilo de sutura de 

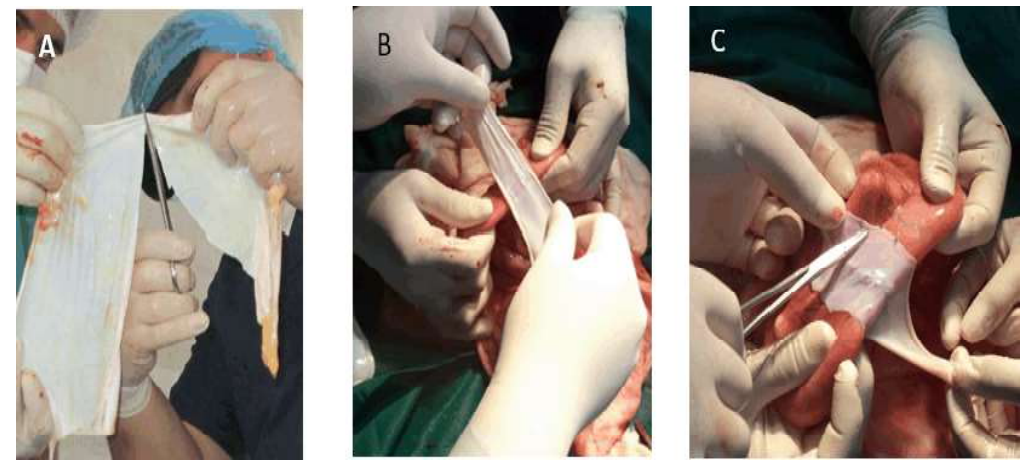

Figura 5. Revestimiento con xenoinjerto de pericardio bovino sobre la línea de sutura anastomótica fijado con suturas simples discontinuas de ácido poliglicólico. (A) Recorte de pericardio bovino de $2.4 \mathrm{~cm}$ aproximadamente; (B y C) Aplicación y fijación del revestimiento de pericardio bovino sobre la serosa yeyunal con puntos simples discontinuos (solo en capa serosa y muscular - no perforante)
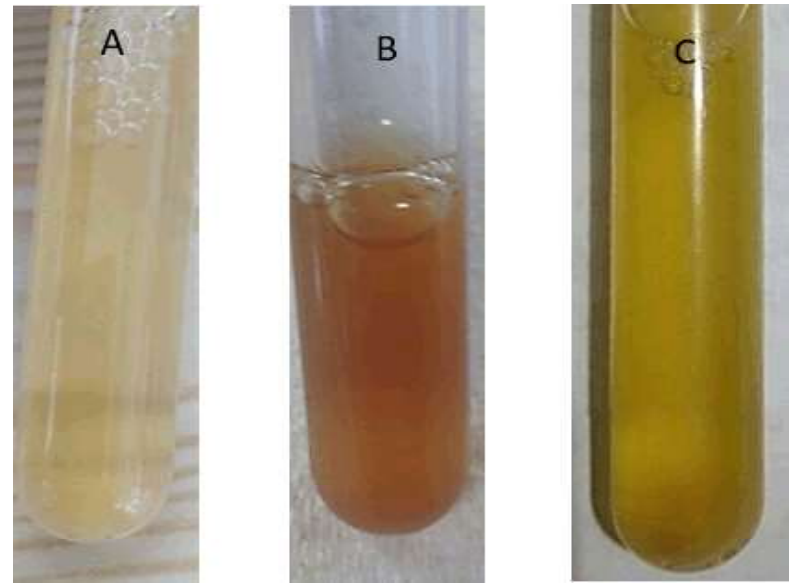

Figura 6. Color y turbidez del líquido peritoneal en caballos con xenoinjerto de pericardio bovino. (A) Muestra preoperatoria; (B) A los 4 días; (C) A los 8 días de la cirugía

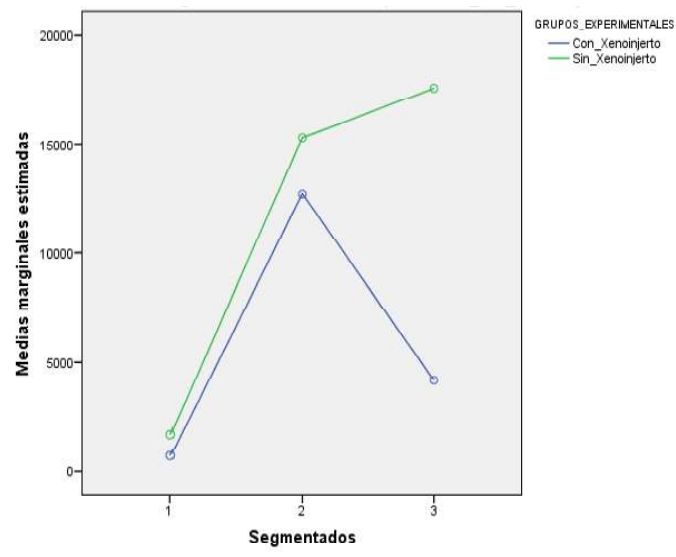

Figura 7. Perfil obtenido en el análisis de varianza factorial para medidas repetidas del recuento total de neutrófilos segmentados. 1,2 y 3 : día 0,4 y 8 posoperatorio, respectivamente. Se observa diferencia significativa entre el día 0 con los días 4 y 8 del posoperatorio 

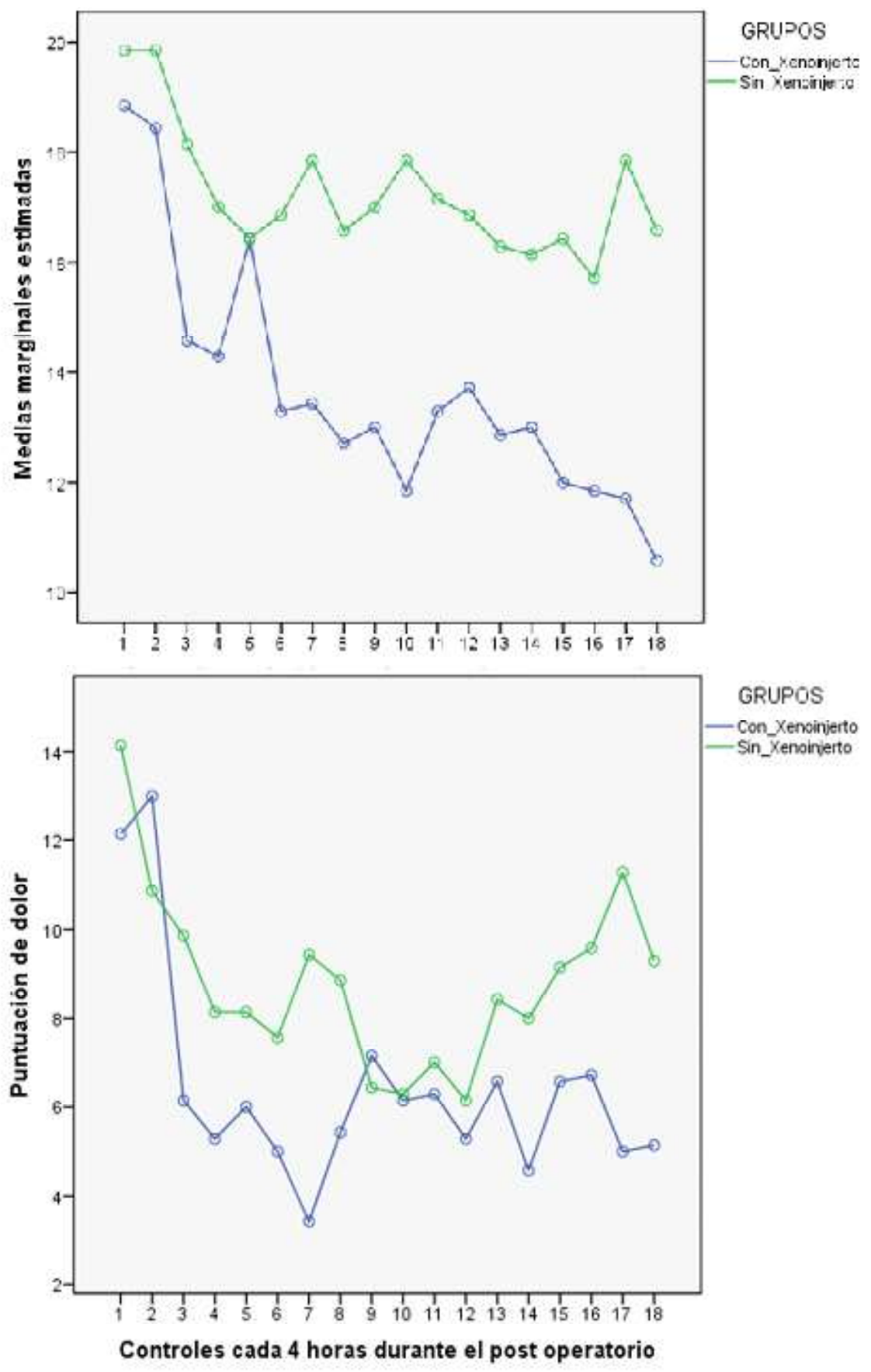

Figura 8. Perfiles obtenidos en el análisis de varianza factorial para medidas repetidas de la evaluación de la influencia del xenoinjerto de pericardio bovino no tratado en el dolor posoperatorio evaluado según la escala de evaluación numérica compuesta (NRS) $(p<0.05)$ y la escala de puntuación de dolor compuesto (CPS) $(p>0.05)$ 
ácido poliglicólico $\mathrm{N} .^{\circ} 1$. La dermis se afrontó con una sutura intradérmica a lo que se sumó una sutura Reverdin en piel con nylon de $0.4 \mathrm{~mm}$.

\section{Técnica quirúrgica para el grupo $A$}

Se empleo la anastomosis término-terminal con xenoinjerto de pericardio bovino descrita para el grupo B, pero además se procedió a revestir la línea de sutura con xenoinjerto de pericardio bovino no tratado, adaptando lo reportado por Testini (2014), utilizando de 8 a 10 puntos de sutura simples discontinuas para fijarlo en la línea de sutura de la anastomosis (Figuras 4 y 5). Una consideración importante radicó en colocar en contacto la serosa del intestino con la cara externa del pericardio, que anatómicamente tiene adherida a la grasa (la cual fue extraída previamente).

El xenoinjerto pericárdico fibroso se obtuvo de bovinos menores a 6 años de un centro de beneficio, antes de que las vísceras torácicas pierdan su condición de limpieza fisiológica y sin recibir tratamiento previo. El tejido fue trasportado en suero fisiológico para usarse en la línea de sutura anastomótica.

\section{Tratamiento Posoperatorio}

Los caballos, luego de la cirugía, fueron albergados en pesebreras independientes de $4 \times 3 \mathrm{~m}$, donde recibieron analgesia en base a tramadol $(2 \mathrm{mg} / \mathrm{kg})$, lidocaína $(0.2 \mathrm{mg} / \mathrm{kg})$, ketamina $(0.5 \mathrm{mg} / \mathrm{kg})$ y xilazina $(0.2 \mathrm{mg} / \mathrm{kg})$ diluido en $1 \mathrm{~L}$ de solución polielectrolítica, aplicada en infusión continua IV a dosis respuesta. Se agregó gentamicina a $6.6 \mathrm{mg} / \mathrm{kg}$ vía IM más penicilina procaínica a $22000 \mathrm{UI} / \mathrm{kg}$ vía IM, cada $12 \mathrm{~h}$. La fluidoterapia se efectuó de acuerdo con los requerimientos y fórmulas fisiológicas estándares.

\section{Alimentación de Soporte}

A partir de las $24 \mathrm{~h}$ de la intervención quirúrgica, se empezó con una rigurosa dieta blanda a base de hojas de alfalfa fresca de manera espaciada, las cuales según Freeman (2012) son bien toleradas. Esta alimentación tiene el beneficio de estimular el proceso de recuperación de la función intestinal. Esto se repitió a intervalos de 3-4 h, aumentando la cantidad en forma progresiva hasta llegar a una ración completa al tercer-cuarto día del posoperatorio.

\section{Cuantificación del Dolor Posoperatorio}

Los signos de cólico posoperatorio se midieron mediante la escala de puntuación de dolor compuesto (CPS) con un rango de 0 a 39 (Bussières et al., 2008) y la escala de evaluación numérica compuesta (NRS) con un rango de 9 a 36 (Pritchett et al., 2003), tomando como base el estudio de van Loon et al. (2014). Debido a limitaciones prácticas, las puntuaciones de dolor fueron realizadas por tres observadores familiarizados con los parámetros de evaluación en caballos libres de dolor, los cuales asignaron los puntajes CPS y NRS por consenso después de $8 \mathrm{~min}$ de evaluación en su respectivo pesebre. Las puntuaciones de dolor se realizaron cada $4 \mathrm{~h}$ durante 3 días del posoperatorio de la anastomosis yeyunal. Los observadores no estaban cegados para el diagnóstico clínico.

El análisis estadístico se hizo mediante un análisis de varianza factorial para medidas repetidas con el fin de distinguir la variabilidad entre las cuantificaciones del dolor en las dos escalas y comparar los resultados de los grupos experimentales, realizando múltiples comparaciones por pares con el ajuste de Bonferroni, utilizando el programa estadístico SPSS v. 22.

\section{Resultados}

Debido a fallas en el transporte de las muestras de líquido peritoneal, solo se analizaron las muestras de seis animales por grupo experimental. En general, todas las muestras fueron inodoras a los 0,4 y 8 días de su colección, mientras que el color varió de ama- 
rillo pajizo en el día 0 a amarillo turbio en el día 4 y amarillo en el día 8 (Figura 6).

En el análisis del recuento total de células nucleadas (RTCN) del grupo con xenoinjerto de pericardio bovino no se hallaron diferencias significativas entre los valores del día 0,4 y 8 posoperatorio ( 0 días: media \pm desviación estándar $=1585 \pm 1118 ; 4$ días: $5975 \pm 11177$; 8 días: $5990 \pm 2636$ ). Asimismo, en el grupo sin xenoinjerto de pericardio bovino tampoco se hallaron diferencias significativas entre los valores del día 0,4 y 8 posoperatorio (0 días: $4580 \pm 7222$; 4 días: $20371 \pm 18586 ; 8$ días: $12429 \pm 9039)$.

Al comparar los resultados del RTCN entre los dos grupos experimentales, no se hallaron diferencias significativas en el día 0 entre el grupo con $(1585 \pm 1118$, $)$ y $\sin$ xenoinjerto ( $4580 \pm 7222)$, en el día 4 entre el grupo con $(15975 \pm 11177)$ y sin xenoinjerto (20371 \pm 18586$)$ y en el día 8 entre el grupo con $(5990 \pm 2636)$ y $\sin$ xenoinjerto $(12429 \pm$ 9039).

En el grupo sin xenoinjerto de pericardio bovino se hallaron diferencias significativas entre los neutrófilos segmentados del preoperatorio $(1661 \pm 2112)$ con aquellos en el día $4(15302 \pm 13507)$ y día 8 del posoperatorio (17572 \pm 17996 ) (Figura 7). Por otro lado, no se hallaron diferencias significativas entre días posoperatorios en las células nucleadas específicas (linfocitos, abastonados, segmentados, monocitos, eosinófilos, basófilos y macrófagos.

Con relación a la cuantificación del dolor posoperatorio de la cirugía experimental, evaluado con tablas NRS, se encontró que el grupo sin xenoinjerto expresó más dolor, evidenciando que el revestimiento de la línea de sutura con xenoinjerto disminuye considerablemente el dolor posoperatorio, especialmente en los controles a las $28,32,36,40,44,68$ y 72 horas $(\mathrm{p}<0.05)$. Asimismo, la del dolor posoperatorio, evaluado con tablas CPS, se encontraron respuestas similares a la tabla
NRS, aunque las diferencias significativas se encontraron específicamente en los controles a las 16,28 y 56 horas del posoperatorio $(\mathrm{p}<0.05)$ (Figura 8).

Cabe resaltar que 13 equinos de los dos grupos experimentales sobrellevaron el posoperatorio evaluado hasta los 100 días, dada la ausencia de signos de cólico y con alimentación normal. El equino restante del grupo A fue sometido a una segunda laparotomía para una evaluación macroscópica fuera del presente estudio, por lo que no se pudo hacer el seguimiento del mismo modo.

\section{Discusión}

La turbidez se incrementó a los cuatro días del posoperatorio, pero regresó a su apariencia normal a los 8 días en ambos grupos experimentales. Si se considera que la densidad o turbidez del líquido peritoneal es la expresión de la alta celularidad, posibles bacterias, fibrina y lípidos, entre otros (Matthews et al., 2002; Dempsey y Ewing, 2011; Lastimer, 2011; Sirois, 2015), se puede concluir que las intervenciones quirúrgicas influyeron temporalmente en el incremento celular y bioquímico del líquido peritoneal.

El RTCN y el recuento de linfocitos se comportaron del mismo modo en los dos grupos experimentales, no hallándose diferencias significativas entre sujetos. Las técnicas quirúrgicas empleadas fueron efectivas, a diferencia de los resultados logrados por Trigo y Valero (2004) y Tizard (2013), quienes mencionan que en el caso de xenoinjertos se desencadena una hipersensibilidad tipo IV. Asimismo, Guyton y Hall (2011) indican que con xenoinjertos ocurren reacciones inmunitarias que provocan la muerte de las células del injerto dentro de las cinco semanas del trasplante, a menos que se emplee un tratamiento específico para evitar las reacciones inmunitarias. 
Los neutrófilos abastonados, específicamente en el grupo experimental sin xenoinjerto, se incrementaron ( $\mathrm{p}<0.057)$ (Figura 7), indicando que el xenoinjerto evita una probable flogosis y fugas de contenido intestinal, dado que una inflamación se presenta con incremento de neutrófilos inmaduros (Núñez y Bouda, 2007). Esto permite inferir que el grupo con xenoinjerto, al no presentar neutrofilia, facilitaría la síntesis de colágeno evitando fugas del contenido intestinal después de la sutura (Grimes et al., 2013).

La sobrevivencia de los animales en los grupos experimentales fue total, lo que resalta la viabilidad de la técnica quirúrgica de anastomosis término-terminal yeyunal con un solo patrón de sutura simple discontinuo de tres capas: serosa, muscular y sub mucosa (Figuras 2 y 3 ). Se entiende que las suturas que provocan eversión o inversión retrasan la cicatrización del intestino pudiendo provocar la formación de estenosis (Fossum, 2009). Asimismo, la tasa de mortalidad y presencia de cólicos en el posoperatorio son mayores en casos de anastomosis latero-lateral (Proudman et al., 2007). Por otro lado, Davis y Rivadeneira (2013) señalan que cuando la saturación de oxígeno disminuye a menos del $30 \%$ en la línea de sutura, la necrosis de la anastomosis ocurre dentro de 48 horas.

El tratamiento posoperatorio tomó en cuenta los problemas asociados al íleo posoperatorio donde los macrófagos intestinales liberan citosinas y óxido nítrico que causan inhibición de la motilidad intestinal (Doherty, 2009; Fleming y Mueller, 2011; Alonso et al., 2014), más los trastornos abdominales propios de la cirugía (Hardy y Rakestraw, 2002; Hackett y Hassel, 2008). Fue así que el tratamiento posoperatorio incluyó el uso de antibióticos, analgésicos y antinflamatorios. La inclusión de lidocaína como agente procinético evita el íleo y previene las adherencias (Driessen, 2013; Alonso et al., 2014), y la xilazina actúa como analgésico visceral (Divers, 2014). No se usaron analgésicos no esteroides inhibidores de la ciclooxigenasa 2 (COX-2), porque reducen la actividad contráctil del intestino delgado, lo cual podría empeorar el íleo propio de la cirugía entérica (Menozzi et al., 2012).

El yeyuno es muy elástico, pero trabaja con poco contenido luminal a flujo constante. La técnica quirúrgica empleada permite un lumen mucho más grande que el que tiene el intestino durante el peristaltismo normal, debido a los $50^{\circ}$ de angulación del corte, como lo indica Freeman (2012), lo cual generó una conformación en forma de «V» de la zona anastomótica (Figura 2), para evitar complicaciones durante una posible contracción cicatricial. Sin embargo, es necesario resaltar que esta forma de «V» desaparece antes de los 100 días de la cirugía, aunque la luz intestinal de la zona anastomótica con el xenoinjerto se observa aún más grande en comparación al resto del intestino (Figura 4), lo que garantiza el tránsito intestinal, dado que no se evidencia alteraciones en el peristaltismo.

En el manejo del pericardio bovino como xenoinjerto se deben tomar las precauciones necesarias de esterilidad, debido a que puede ser una fuente potencial de transmisión de patógenos (Santos et al., 2013).

El dolor posoperatorio en el grupo de caballos con xenoinjerto de pericardio bovino estuvo significativamente disminuido en la evaluación con la escala NRS, aunque no marcadamente con la escala CPS. No obstante, se observan las ventajas de la técnica quirúrgica con el xenoinjerto, al encontrarse una menor concentración neutrofílica de segmentados y abastonados en el líquido peritoneal en este grupo. Nuñez y Bouda (2007) señalan que la desaparición de la desviación a la izquierda, la disminución de neutrófilos tóxicos y la recuperación del número de linfocitos y de eosinófilos son indicadores del control de la inflamación.

Se reporta mayor fiabilidad entre observadores al puntuar la CPS en comparación a la NRS, al considerar que el uso de la CPS mejora la objetividad de la puntuación del dolor 
en caballos después de la cirugía gastrointestinal (van Loon et al., 2014). De ser así, se podría decir que el xenoinjerto utilizado, en realidad, no influye en el dolor posoperatorio, sino que evita posibles fallas anastomóticas que podrían ser dolorosas y críticas.

Según los resultados de este estudio, el xenoinjerto de pericardio bovino no tratado es una buena opción para reforzar la anastomosis del intestino delgado en el equino, debido a que no influye en las concentraciones celulares del líquido peritoneal ni en el dolor posoperatorio. No obstante, estos resultados difieren de otros reportes que describen que el refuerzo con vellones de colágeno tratado puede causar obstrucción intestinal (Hoeppner et al., 2009; Schreinemacher et al., 2011).

Es importante indicar que los resultados obtenidos se circunscriben a las características de este estudio. Kotze et al. (2013) exponen claramente las condiciones del pronóstico, mencionando que la predicción de la dehiscencia anastomótica no es posible por la influencia de factores relacionados con el paciente, como edad, sexo, nivel de albuminemia, uso de corticosteroides y el estado ASA. Asimismo, se tienen elementos relacionados con el procedimiento quirúrgico (la tensión en el lugar de la anastomosis, el suministro de sangre, la duración de la cirugía) y la condición intestinal (enfermedad inflamatoria, neoplasias, metástasis y peritonitis que también juegan un papel preponderante en la curación anastomótica) (Davis y Rivadeneira, 2013).

\section{Conclusión}

El xenoinjerto de pericardio bovino no tratado, como revestimiento de la anastomosis término-terminal yeyunal equina no altera el color, la turbidez, el olor ni el recuento total de células nucleadas del líquido peritoneal, ni agudiza el cólico posoperatorio; lo que refuerza el proceso de remodelación cicatricial, evitando posibles fugas anastomóticas y peritonitis.

\section{Literatura Citada}

1. Abutarbush S, Carmalt J, Shoemaker R. 2005. Causes of gastrointestinal colic in horses in western Canada: 604 cases (1992 to 2002). Can Vet 46: 800-805.

2. Alonso JM, Garcia Alves AL, Watanabe MJ, Rodrigues CA, Hussni $C A$. 2014. Peritoneal response to abdominal surgery: the role of equine abdominal adhesions and current prophylactic strategies. Vet Med Int 2014: ID 279730. doi: 10.1155/2014/27973

3. Al-Rukibat RK, Ismail ZB, Al-Majali AM, Al-Zghoul MB. 2006. Peritoneal fluid analysis in adult, nonpregnant Awassi sheep. Vet Clin Pathol 35: 215218.

4. Astarcioçlu H, Koçdor MA, Sökmen S, Karademir S, Özer E, Bora S. 2001. Comparison of different surgical repairs in the treatment of experimental duodenal injuries. Am J Surg 181: 309-312. doi: 10.1016/S0002-9610(01)00586-4

5. Bladon BM, Hillyer MH. 2000. Effect of extensive ileal resection with a large resulting mesenteric defect and stapled ileal stump in horses with a jejunocaecostomy: a comparison with other anastomotic techniques. Equine Vet J Suppl 32: 52-58. doi: 10.1111/j.20423306.2000.tb05334.x

6. Bussières $G$, Jacques $C$, Lainay $O$, Beauchamp G, Leblond A, Cadoré JL, Desmaizières L-M, et al. 2008. Development of a composite orthopaedic pain scale in horses. Res Vet Sci 85: 294306. doi: 10.1016/j.rvsc.2007.10.011

7. Claunch K, Mueller P. 2012. Treating intra-abdominal adhesions: the surgeon's dilemma. Equine Vet Educ 24: 552-555. doi: $10.1111 / j .2042-3292.2012 .00438 . x$ 
8. Davis B, Rivadeneira DE. 2013. Complications of colorectal anastomoses: leaks, strictures, and bleeding. Surg Clin North Am 93: 61-87. doi: 10.1016/ j.suc.2012.09.014

9. Dempsey SM, Ewing PJ. 2011. A review of the pathophysiology, classification, and analysis of canine and feline cavitary effusions. J Am Anim Hosp Assoc 47: 1-11. doi: 10.5326/ JAAHA-MS-5558

10. Divers T. 2014. Anaesthesia of the horse. In: Veterinary anaesthesia. $11^{\text {th }} \mathrm{ed}$. China: Elsevier. p 245-311.

11. Doherty TJ. 2009. Postoperative ileus: pathogenesis and treatment. Vet Clin North Am Equine Pract 25: 351-362. doi: 10.1016/j.cveq.2009.04.011

12. Driessen B. 2013. Pain management. In: Equine emergencies. $4^{\text {th }}$ ed. Canada: Elsevier. p 749-763.

13. Fleming K, Mueller E. 2011. Ileal impaction in 245 horses: 1995-2007. Can J Vet 52: 759-763.

14. Fossum TW. 2009. Cirugía de pequeños animales. $3^{\circ}$ ed. España: Elsevier. $1632 \mathrm{p}$.

15. Freeman D. 2012. Small intestine. In: Equine surgery. $4^{\text {th }}$ ed. Canada: Elsevier. p 416-453.

16. Gelberg HB. 2014. Comparative anatomy, physiology, and mechanisms of disease production of the esophagus, stomach, and small intestine. Toxicol Pathol 42: 54-66. doi: 10.1177/ 0192623313518113

17. Grimes J, Schmiedt C, Milovancev M, Radlinsky M, Cornell K. 2013. Efficacy of serosal patching in dogs with septic peritonitis. J Am Anim Hosp Assoc 49: 246-249. doi: 10.5326/JAAHA-MS-5870

18. Guyton A, Hall J. 2011. Tratado de fisiología médica. $11^{\circ}$ ed. España: Elsevier. $1115 \mathrm{p}$.

19. Hackett E, Hassel D. 2008. Colic: nonsurgical complications. Vet Clinic Equine Pract 24: 535-555.

20. Hansen L, Monnet E. 2013. Evaluation of serosal patch supplementation of surgical anastomoses in intestinal segments from canine cadavers. Am J Vet Res 74: 1138-1141. doi: 10.2460/ ajvr.74.8.1138

21. Hardy J, Rakestraw PC. 2002. Postoperative management for colics. Clin Tech Equine Pract 1: 188-197. doi: 10.1053/ctep.2002.35580

22. Hoeppner J, Crnogorac V, Marjanovic G, Jüttner E, Keck T, Weiser H-F, Hopt UT. 2009. Small intestinal submucosa for reinforcement of colonic anastomosis. Int J Colorectal Dis 24: 543-550.

23. König H, Liebich H. 2005. Anatomía de los animales domésticos: órganos, sistema circulatorio y sistema nervioso. $2^{\circ}$ ed. Madrid: Ed Médica Panamericana. $381 \mathrm{p}$.

24. Kotze PG, Barcelos IF De, Ropelato $R V$, Coy CSR. 2013. Human fibrinogen and thrombin patch for extraluminal protection of intestinal anastomosis. $\mathrm{J}$ Coloproctol 33: 174-178. doi: 10.1016/ j.jcol.2013.08.004

25. Lastimer K. 2011. Duncan and Prase's veterinary laboratory medicine: clinical pathology. USA: John Wiley \&Sons. 524p.

26. Li L, Wang $N$, Jin $X$, Deng $R$, Nie S, Sun L, Wu Q, Wei Y, Gong C. 2014. Biodegradable and injectable in situ cross-linking chitosan-hyaluronic acid based hydrogels for postoperative adhesion prevention. Biomaterials 35: 3903-3917. doi: 10.1016/j.biomaterials.2014.01.050

27. Lorello O, Orsini JA. 2014. Gastrointestinal system. In: Equine emergencies. $4^{\text {th }}$ ed. Canada: Elsevier. p 157-237.

28. Marshall JF, Blikslager AT. 2012. Colic: diagnosis, surgical decision, and preoperative management. In: Auer J, Stick J (eds.) Equine Surgery. The Netherlands: Elsevier. p 402-407.

29. Matthews S, Dart A, Reid S, Dowling B, Hodgson D. 2002. Predictive values, sesitivity and specificity of abdominal fluid variables in determining the neef for surgery in horses with an acute abdominal crisis. Aust Vet J 80: 132-136. doi: 10.1111/j.1751-0813.2002.tb11372.x 
30. Mcgowan CM, Hodgson DR. 2014. Hematology and biochemistry. In: The athletic horse. Principles and practice of equine sports medicine. $2^{\text {nd }}$ ed. China: Elsevier. p 56-68.

31. Menozzi A, Pozzoli C, Zullian C, Poli E, Serventi P, Bertini S. 2012. Inhibition of motility in isolated horse small intestine is mediated by $\mathrm{k}$ but not $\mu$ opioid receptors. Equine Vet J 44: 368370. doi: 10.1111/j.2042-3306.2011.00426.x

32. Nazar C, Zamora M, González A. 2015. Cirugía ambulatoria: selección de pacientes y procedimientos quirúrgicos. Rev Chil Cir 67: 207-213. doi: 10.4067/ S0718-40262015000200017

33. Nuñez L, Bouda J. 2007. Patología clínica veterinaria. $2^{\circ}$ ed. Chávez A (ed). México: Universidad Autónoma de México. $334 \mathrm{p}$.

34. Polak R, Pitombo RNM. 2011. Care during freeze-drying of bovine pericardium tissue to be used as a biomaterial: a comparative study. Cryobiology 63: 61-66. doi: 10.1016/ j.cryobiol.2011.05.001

35. Pritchett LC, Ulibarri C, Roberts MC, Schneider RK, Sellon DC. 2003. Identification of potential physiological and behavioral indicators of postoperative pain in horses after exploratory celiotomy for colic. Appl Anim Behav Sci 80: 31-43. doi: 10.1016/S01681591(02)00205-8

36. Proudman CJ, Edwards GB, Barnes J. 2007. Differential survival in horses requiring end-to-end jejunojejunal anastomosis compared to those requiring sideto-side jejunocaecal anastomosis. Equine Vet J 39: 181-185. doi: 10.2746/ $042516407 X 171354$

37. Santos MH, Silva RM, Dumont VC, Neves JS, Mansur HS, Heneine LGD. 2013. Extraction and characterization of highly purified collagen from bovine pericardium for potential bioengineering applications. Materials Sci Engineering C 33: 790-800. doi: 10.1016/ j.msec.2012.11.003
38. Schreinemacher MH, Bloemen JG, van der Heijden SJ, Gijbels MJ, Dejong CH, Bouvy ND. 2011. Collagen fleeces do not improve colonic anastomotic strength but increase bowel obstructions in an experimental rat model. Int J Colorectal Dis 26: 729-735. doi: 10.1007/s00384-011-1158-z

39. Schwarz BC, van den Hoven $R$, Schwendenwein I. 2012. Diagnostic value of the neutrophil myeloperoxidase index in horses with systemic inflammation. Vet J 191: 72-78. doi: 10.1016/j.tvj1.2010.12.010

40. Siobhan M. 2014. Gastrointestinal system. In: Knottenbelt and Pascoe's color atlas of diseases and disorders of the horse. $2^{\text {nd }}$ ed. China: Elsevier. p 1-83.

41. Sirois M. 2015. Laboratory procedures for veterinary technicians. $6^{\text {th }}$ ed. China: Elsevier. $440 \mathrm{p}$.

42. Smith CL, Dowling BA, Dart AJ. 2005. Recent advances in equine abdominal surgery. Vet J 170: 41-51. doi: 10.1016/j.tvj1.2004.03.019

43. Stewart S, Southwood L, Aceto H. 2014. Comparison of short and long term complications and survival following jejunojejunostomy, jejunoileostomy and jejunocaecostomy in 112 horses: 20052010. Equine Vet J 46: 333-338. doi: 10.1111/evj.12143

44. Telem D, Chin E, Nguyen S, Divino C. 2010. Risk factors for anastomotic leak following colorectal surgery: a casecontrol study. Arch Surg 145: 371-376. doi: 10.1001/archsurg.2010.40

45. Testini M, Gurrado A, Portincasa P, Scacco S, Marzullo A, Piccinni G, Lissidini G, et al. 2014. Bovine pericardium patch wrapping intestinal anastomosis improves healing process and prevents leakage in a pig model. PloS One 9(1): e86627. doi: 10.1371/ journal.pone.0086627

46. Tizard I. 2013. Veterinary immunology. $9^{\text {th }}$ ed. China: Saunders. 552 p.

47. Trigo F, Valero G. 2004. Patología general veterinaria. $4^{\circ}$ ed. México: Universidad Autónoma de México. 437 p. 
48. van den Boom $R$, van der Velden MA. 2001. Surgery: short- and long-term evaluation of surgical treatment of strangulating obstructions of the small intestine in horses: a review of 224 cases. Vet Q 23: 109-115. doi: 10.1080/ 01652176.2001 .9695095
49. van Loon J, Jonckheer-Sheehy V, Back W, van Weeren R, Hellebrekers L. 2014. Monitoring equine visceral pain with a composite pain scale score and correlation with survival after emergency gastrointestinal surgery. Vet J 200: 109115. doi: 10.1016/j.tvj1.2014.01.003 